\title{
Comparison of Molecular Species Distribution of DHA-containing Triacylglycerols in Milk and Different Infant Formulas by Liquid Chromatography-Mass Spectrometry
}

Zhiqian Liu ${ }^{1}$, Benjamin G. Cocks ${ }^{1,2}$ and Simone Rochfort ${ }^{1,2}$

${ }^{1}$ Biosciences Research, Department of Economic Development, Jobs, Transport and Resources, AgriBio, 5 Ring Road, Bundoora, Victoria 3083, Australia,

${ }^{2}$ School of Applied Systems Biology, La Trobe University, Bundoora, Victoria 3083, Australia

Corresponding author:

Email: simone.rochfort@ecodev.vic.gov.au

Phone: +61-3-9032 7110

Fax: +61-3-9032 7601

Running title: DHA-containing triacylglycerols in milk and infant formulas 


\section{Lipid extraction}

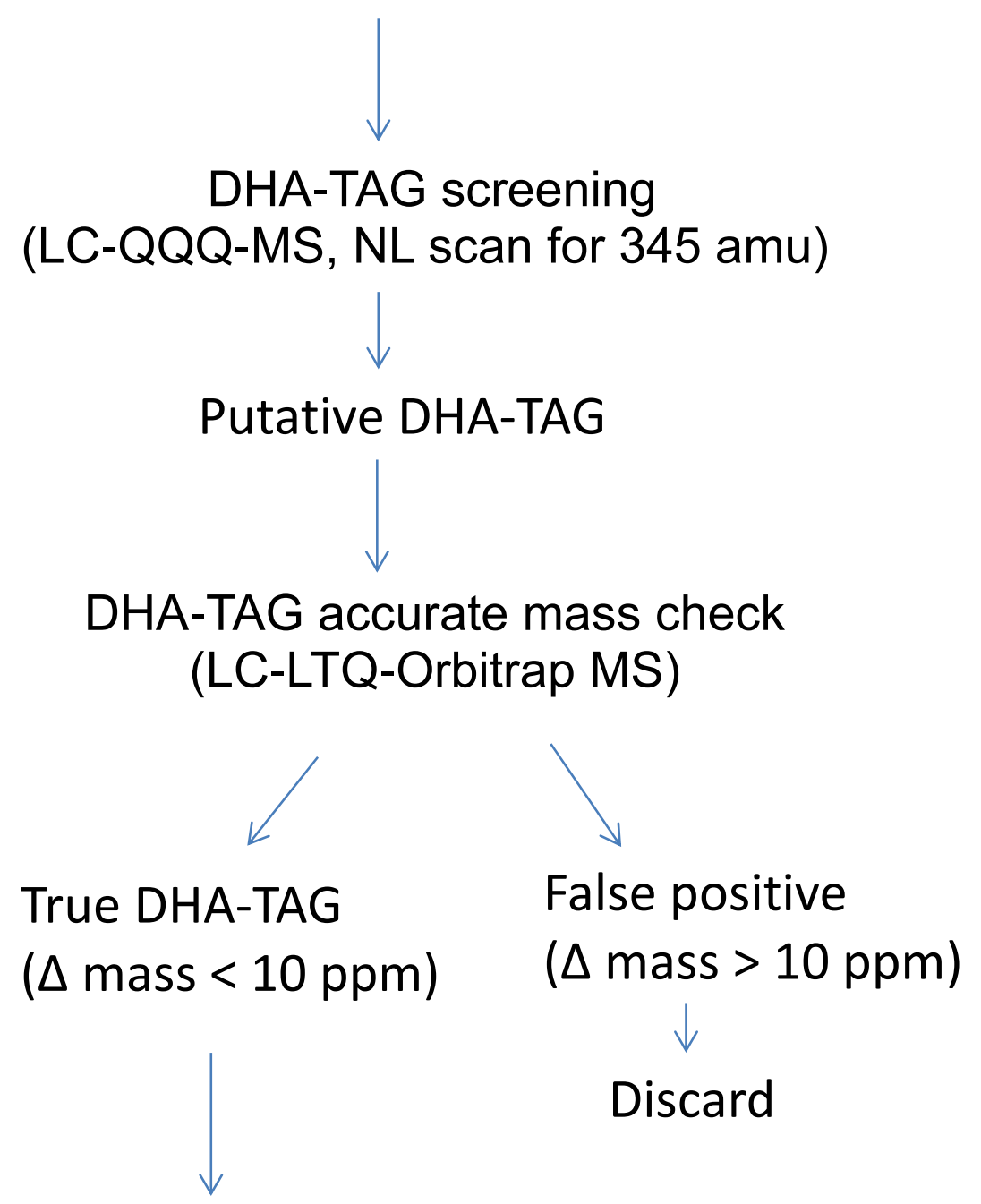

DHA-TAG fatty acid composition determination

(LC-LTQ-Orbitrap MS/MS)

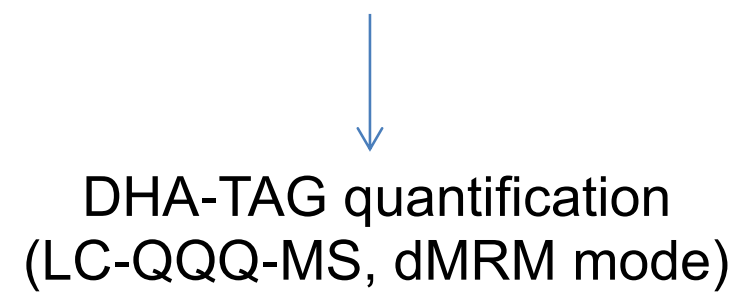

Figure S-1. A schematic workflow for identification and quantification of DHA-TAG in milk and infant formula samples. 


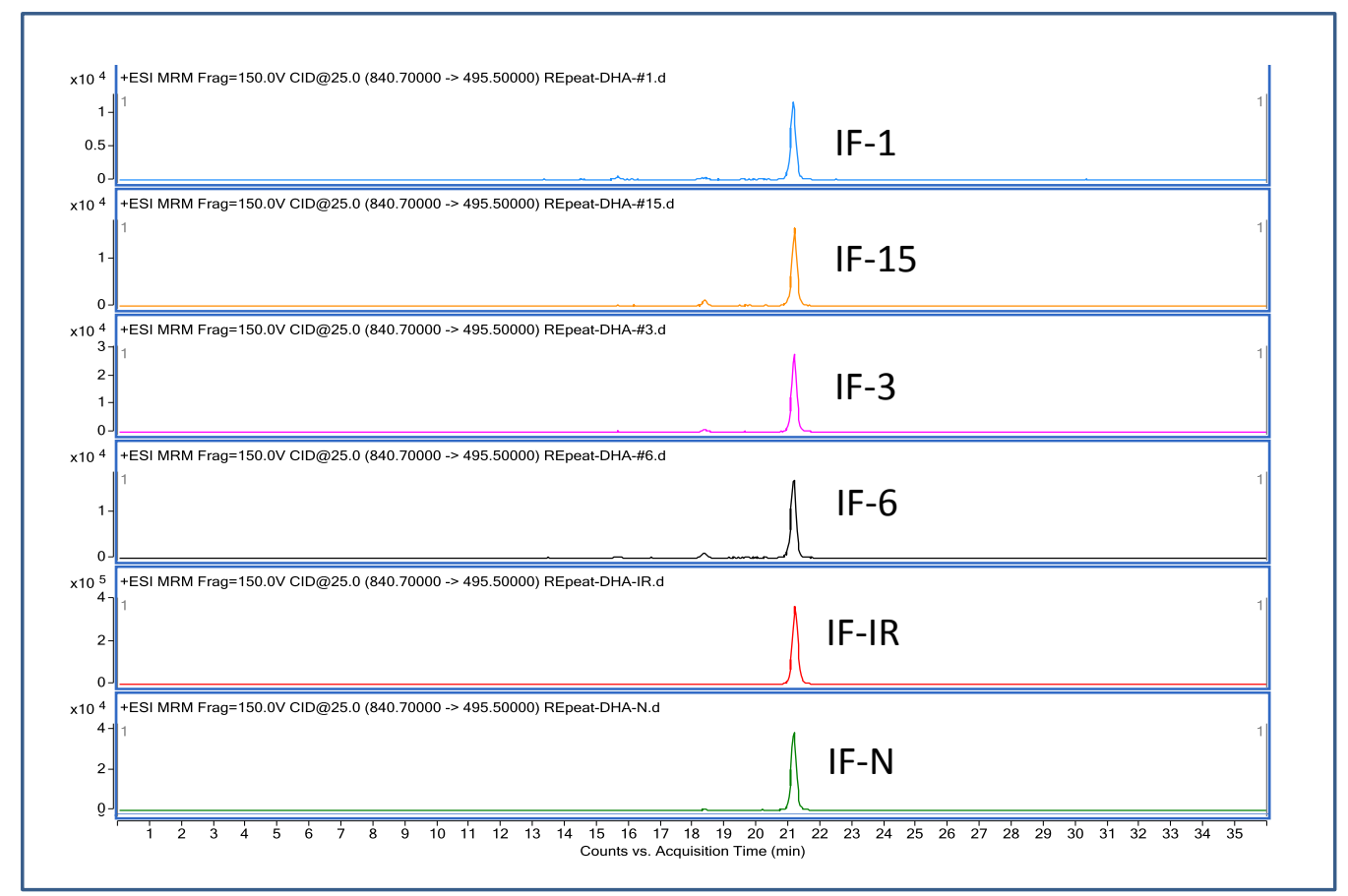

Figure S-2. LC-Triple Quadrupole MS profile of the specific product ion $(\mathrm{m} / \mathrm{z}, 495.5)$ of DHA-7 detected in different IFs in MRM mode. 


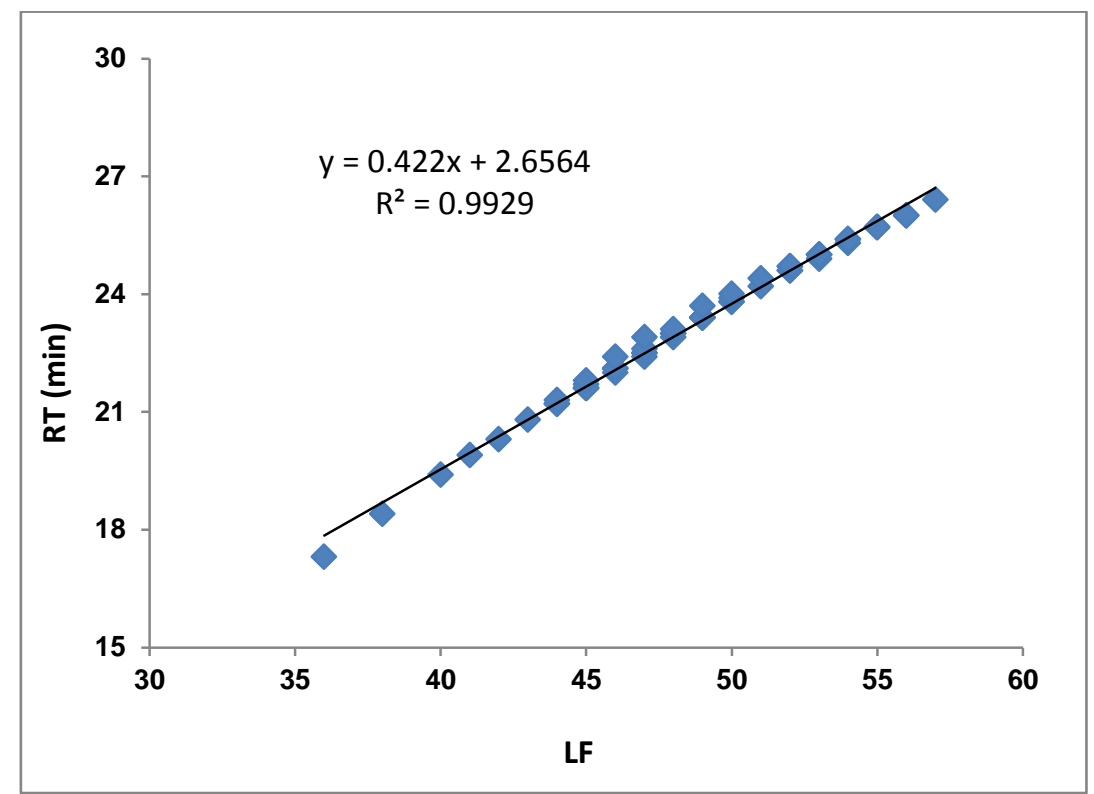

Figure S-3. Correlation between retention time (RT) and lipophilicity factor (LF) of DHATAG. LF: total carbon number - double bond number. 


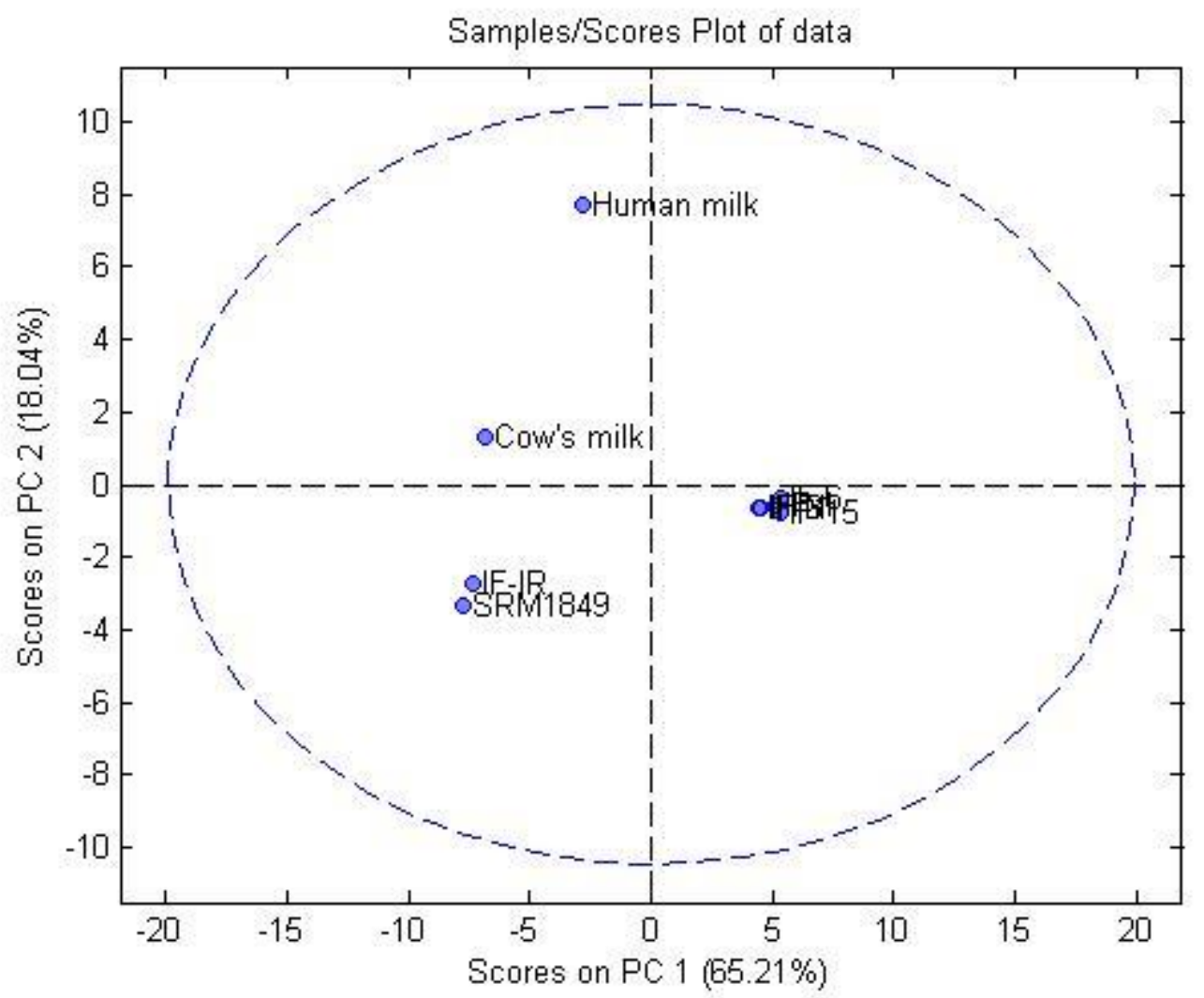

Figure S-4. PCA plot of the nine samples based on their DHA-TAG composition. 